\title{
ORTHOGONAL GROUPS OVER LOCAL RINGS
}

\author{
BY WILHELM KLINGENBERG
}

Communicated by J. Dieudonné, October 28, 1960

In an earlier paper [5] we have determined the structure of the linear groups over a local ring. In this note we continue the study of the classical groups over a local ring with the investigation of the orthogonal groups.

Our main result (cf. Theorem 6 below) is a complete description of the invariant subgroups of an orthogonal group of noncompact type (i.e., of index $\geqq 1$ ) over a local ring $L$ of characteristic $\neq 2$. Certain low dimensional cases being excluded, the result reads as follows: The set of invariant subgroups splits into disjoint classes $\mathbb{C}(J)$ which are in one-to-one correspondence with the ideals $J$ of $L$. Each class has a greatest and a smallest element, with respect to the inclusion, which are represented by certain congruence subgroups modulo $J$, and every group between the greatest and the smallest element of $\mathfrak{e}(J)$ belongs to $\mathfrak{e}(J)$.

A similar result does hold for the set of invariant subgroups of the commutator group of the orthogonal group; in this case, the structure of the classes $\mathfrak{e}(J)$ is very simple since each class contains at most two elements, and then the smaller element has index 2 in the greater one.

Hence, it turns out that the structure of the orthogonal groups under consideration is of the same type as the structure of the linear groups over a local ring, cf. [5]: Here too the invariant subgroups split into classes which correspond to the ideals of the local ring, and each class has a greatest and a smallest element, represented by certain congruence subgroups, and each group in between belongs to the class. One may expect, therefore, that this is the typical arrangement of the invariant subgroups of a classical group over a local ring.

If the local ring $L$ possesses no ideals apart from $L$ and 0 , i.e., if $L$ is a field, then we get the results of Dieudonné $[3 ; 4]$ on the structure of the orthogonal groups over a field.

1. Basic definitions. A local ring is a commutative ring $L$ with unit and a greatest ideal $I \neq L . L^{*}=L-I$ forms a group under the multiplication. The homomorphic image of a local ring, if it is not the zero ring, is again a local ring. $L / I$ is a field. We assume: $\operatorname{char}(L / I) \neq 2$.

An (n-dimensional) vector space over $L$ is an $L$-module isomorphic to $L^{n}$. Let $\Phi$ be a symmetric bilinear form on a vector space. $\Phi$ deter- 
mines an homomorphism $g_{\Phi}$ of the vector space into its dual, cf. Bourbaki [2]. $\Phi$ is called nondegenerate, if $g_{\Phi}$ is an isomorphism.

A metric vector space (over $L$ ), denoted by $V$ or $V(L)$, is a vector space over $L$ on which there is given a nondegenerate symmetric bilinear form $\Phi$.

A subspace $U$ of $V$ is a submodule of $V$ (considered as $L$-module), with the following properties: (i) $U$ is a direct summand, (ii) ker$\operatorname{nel}\left(g_{\Phi \mid U}: U \rightarrow U^{*}\right)$ is a direct summand.

Note. If $L$ is a field, each submodule of $V$ is a subspace. To get uniform definitions and results we assume: $\operatorname{dim} V \geqq 3$. For a subspace $U$ the orthogonal subspace $U^{o}$ is the submodule annulled by $g_{\Phi} U$. $U^{o}$ is a subspace. We have: $\operatorname{dim} U+\operatorname{dim} U^{o}=\operatorname{dim} V . U^{o o}=U$.

A subspace $U$ is called isotropic, if $\operatorname{kernel}\left(g_{\Phi \mid U}\right) \neq 0$, and totally isotropic, if kernel $\left(g_{\Phi \mid U}\right)=U$. Examples of nonisotropic subspaces (i.e., subspaces $U$ with $\operatorname{kernel}\left(g_{\Phi \mid U}\right)=0$ ) are $V$ and $0=0$-space.

A vector $X \in V$ is called nonisotropic, if the submodule $\langle X\rangle$, generated by $X$, is a nonisotropic subspace $\neq 0 . X$ is called isotropic, if $\langle X\rangle$ is isotropic. A nonisotropic vector $X$ is characterized by the properties: $X \neq 0 \bmod I$ and $\Phi(X, X) \in L^{*}$. An isotropic vector is characterized by: $X \neq 0 \bmod I$ and $\Phi(X, X)=0$.

An isomorphism of a space $V$ into a space $V^{\prime}$ is called isometry.

The group of isometries of $V$ onto $V$ is called orthogonal group of $V, O(V)$. The subgroup of isometries with determinant one is called special orthogonal group of $V, S O(V)$. We have: center $O(V)=\{1,-1\}$. Let $J$ be an ideal of $L$ with $J \subset I$. The natural homomorphism $g_{J}: L \rightarrow L / J$ determines a homomorphism (also denoted by $g_{J}$ )

$$
g_{J}: V(L) \rightarrow V(L / J),
$$

where $V(L / J)$ is a space over the local ring $L / J$ with a nondegenerate symmetric bilinear form $h_{J} \Phi$ characterized by: $\left(h_{J} \Phi\right)\left(g_{J} X, g_{J} Y\right)$ $=g_{J} \Phi(X, Y)$ for $(X, Y) \in V \times V$. We will permit in (1) also the ideal $J=L$ by putting $V(L / L)=0=0$-space.

The homomorphism $g_{J},(1)$, determines a homomorphism

$$
h_{J}: O(V(L)) \rightarrow O(V(L / J))
$$

with the characteristic property: $h_{J} \sigma g_{J}=g_{J} \sigma$ for $\sigma \in O(V)$. Here $O(V(L / L))$ denotes the unit group $E$.

The congruence subgroup $\bmod J$ of $O(V), O(V, J)$, is the invariant subgroup consisting of the elements $\sigma \in O(V)$ with $h_{J} \sigma \in$ center $O\left(g_{J} V\right)$. $S O(V) \cap O(V, J)$ is called special congruence subgroup mod $J$ of $S O(V)$, notation: $\operatorname{SO}(V, J)$.

Note. $O(V, L)=O(V)$. $S O(V, L)=S O(V) . O(V, O)=$ center $O(V)$. Here $O$ denotes the zero ideal. 
For each ideal $J$ of $L$ the congruence commutator subgroup $\bmod J$ of $\mathbf{S O}(V), \boldsymbol{\Omega}(V, J)$, is defined as the mixed commutator group $\operatorname{comm}(\mathbf{S O}(V), \mathbf{S O}(V, J)) . \boldsymbol{\Omega}(V, L)$, i.e., the commutator group of $\boldsymbol{S O}(V)$, will also be denoted by $\boldsymbol{\Omega}(V)$. Note: $\boldsymbol{\Omega}(V, O)=E$.

2. The theorems of Witt and Cartan-Dieudonné. A first characterization of the congruence commutator groups. We have the following theorem which reduces to the theorem of Witt if $L$ is a field:

Theorem 1. Let $V$ and $V^{\prime}$ be isometric spaces. If $\sigma: U \rightarrow V^{\prime}$ is an isometry of a subspace $U$ of $V$ into $V^{\prime}$, then there exists an isometry of $V$ onto $V^{\prime}$ which is an extension of $\sigma$.

As a consequence we have that all maximal totally isotropic subspaces of $V$ have the same dimension; this dimension is called the index of $V$, notation: ind $V$. We have: 2 ind $V \leqq \operatorname{dim} V$. If $U$ is a nonisotropic subspace, then we have $V=U+U^{o}$ (direct sum). The symmetry with respect to $U$ is the isometry $\sigma \in O(V)$ given by $\sigma \mid U=1$, $\sigma \mid U^{o}=-1$.

Especially important are the symmetries with respect to a (nonisotropic) hyperplane, i.e., a subspace of codimension 1. Generalizing a result of $\mathrm{E}$. Cartan and Dieudonné we have the

TheOREM 2. Each element $\sigma \in O(V)$ is the product of at most $2 n-2$ symmetries with respect to a hyperplane, where $n=\operatorname{dim} V$. If and only if $\sigma$ is in $\mathbf{S O}(V)$, the number of symmetries representing $\sigma$ will be even.

REMARK. If $L$ is field, each $\sigma \in O(V)$ can be written as a product of $\leqq n$ symmetries with respect to a hyperplane (cf. Dieudonné [3]). We have not been able to prove this for a general local ring. As a consequence of Theorem 2 we have that the homomorphism $h_{J}$, (2), is a map onto.

A first characterization of the congruence commutator groups is given by the

TheOREM 3. $\Omega(V, J)$ is being generated by the elements $\left(\tau \tau^{\prime}\right)^{2}$, where $\tau$ and $\tau^{\prime}$ are symmetries with respect to hyperplanes and $h_{J}\left(\tau \tau^{\prime}\right)=1$.

Among the consequences we have $\boldsymbol{\Omega}(V)$ contains the square of each element of $S O(V)$. $S O(V) / \Omega(V)$ is commutative and each element has order $\leqq 2$. The centralizer of $\boldsymbol{\Omega}(V)$ is equal to the centralizer of $S O(V)$ in $O(V)$ and consists of the elements 1 and -1 . center $S O(V)$ $=\boldsymbol{S O}(V) \cap$ center $\boldsymbol{O}(V)$. center $\boldsymbol{\Omega}(V)=\boldsymbol{\Omega}(V) \cap$ center $\boldsymbol{O}(V)$.

3. The Clifford algebra and the spinor norm. A second characterization of the congruence commutator group. The Clifford algebra over $V, C(V)$, is defined in the usual way, cf. Bourbaki [2]. Denote 
by $C^{+}(V)$ the subalgebra of $C(V)$ generated by the products of an even number of vectors. In the multiplicative group of $C^{+}(V)$ we have the special Clifford group, $D(V)$, consisting of the products of an even number of nonisotropic vectors, considered as elements of $C(V) . D(V) / L^{*}$ is canonically isomorphic to $S O(V)$.

On $D(V)$ we have a canonical homomorphism $N$ into the multiplicative group $L^{*}$ (Bourbaki [2] calls this homomorphism spinor norm) which is quadratic in $L^{*}$. Since $S O(V)$ is isomorphic to $D(V) / L^{*}, N$ determines a homomorphism

$$
\theta: \mathrm{SO}(V) \rightarrow L^{*} / L^{* 2}
$$

which we call spinor norm.

For $\sigma \in S O(V), \theta(\sigma)$ is determined as follows: According to Theorem $3, \sigma$ can be represented as the product of an even number of symmetries $\tau_{i}$ with respect to nonisotropic hyperplanes $H_{i}$. For each $i$, choose a nonisotropic vector $A_{i} \in H_{i}^{o}$. Then $\theta(\sigma)=\prod \Phi\left(A_{i}, A_{i}\right) L^{* 2}$.

kernel $(\theta)$ is called reduced orthogonal group over $V, O^{\prime}(V)$. Obviously $\boldsymbol{\Omega}(V) \subset O^{\prime}(V)$.

If ind $V \geqq 1$, then $O^{\prime}(V)=\boldsymbol{\Omega}(V)$ and the spinor norm $\theta$, (3), plays for the orthogonal groups the same role which the determinant plays for the linear groups. In particular, the spinor norm yields a second characterization of the congruence commutator groups:

Theorem 4. Assume ind $V \geqq 1$. Let $J$ be an ideal of $L$ with $J \subset I$. Denote by $\theta_{J}$ the homomorphism

$$
\theta \times h_{J}: \sigma \in \mathrm{SO}(V) \rightarrow\left(\theta(\sigma), h_{J}\right) \in L^{*} / L^{* 2} \times \mathbf{S O}\left(g_{J} V\right),
$$

(i) $\operatorname{kernel}\left(\theta: \mathbf{S O}(V) \rightarrow L^{*} / L^{* 2}\right)=\boldsymbol{\Omega}(V)$

$$
\operatorname{kernel}\left(\theta_{J}: \mathbf{S O}(V) \rightarrow L^{*} / L^{* 2} \times \mathbf{S O}\left(g_{J} V\right)\right)=\boldsymbol{\Omega}(V, J) \text {, }
$$

(ii) $\mathbf{S O}(V) / \boldsymbol{\Omega}(V)$ is isomorphic to image $(\theta)=L^{*} / L^{* 2}$, and $\mathbf{S O}(V, J) / \mathbf{\Omega}(V, J)$ is isomorphic to image $\left(\theta_{J} \mid \mathbf{S O}(V, J)\right)=$ the subgroup of $L^{*} / L^{* 2} \times$ center $\operatorname{SO}\left(g_{J} V\right)$ consisting of the pairs $(\bar{a}, \bar{\sigma})$ with $g_{J} \bar{a}=\theta(\bar{\sigma})$.

(iii) $(\boldsymbol{S O}(V, J) \cap \boldsymbol{\Omega}(V)) / \boldsymbol{\Omega}(V, J)$ is isomorphic to center $\boldsymbol{\Omega}\left(g_{J} V\right)$.

Corollary. (i) kernel $\left(h_{J}: \boldsymbol{\Omega}(V) \rightarrow \boldsymbol{\Omega}\left(g_{J} V\right)\right)=\boldsymbol{\Omega}(V, J)$,

(ii) If $\operatorname{dim} V o d d$, i.e., if center $\mathbf{S O}\left(g_{J} V\right)=1$, then $\mathbf{S O}(V, J) / \Omega(V, J)$ is isomorphic to $g_{J}^{-1}(L / J)^{* 2} / L^{* 2}$,

(iii) If $\operatorname{dim} V$ odd, then center $\boldsymbol{\Omega}\left(g_{J} V\right)=1$ and therefore: $\mathbf{S O}(V, J)$ $\cap \boldsymbol{\Omega}(V)=\boldsymbol{\Omega}(V, J)$.

4. The projective linear groups in 2 variables over a local ring. Let $L$ be a local ring with greatest ideal $I \neq L$. Assume $\operatorname{char}(L / I) \neq 2$ and 
$L / I \neq F_{3}$. In [5] we have defined the general and the special linear group in 2 variables over $L$, denoted by $G L(2, L)$ and $S L(2, L)$, respectively.

For each ideal $J$ of $L$ we have the canonical homomorphism

$$
h_{J}: G L(2, L) \rightarrow G L(2, L / J) \text {. }
$$

Here, $G L(2, L / L)$ denotes the unit group. Using the map $h_{J}$, (4), and the determinant, we have, for each ideal $J$ of $L$, the following two invariant subgroups, cf. [5]:

$G C(2, L, J)=$ group of the $\sigma \in G L(2, L)$ with $h_{J} \sigma \in$ center $G L(2, L / J)$, $S C(2, L, J)=$ group of the $\sigma \in G L(2, L)$ with $h_{J} \sigma=1$ and $\operatorname{det} \sigma=1$.

Note. $G C(2, L, L)=G L(2, L) ; S C(2, L, L)=S L(2, L)$.

Consider the canonical homomorphism

$$
P: G L(2, L) \rightarrow G L(2, L) / \text { center } G L(2, L) .
$$

The image $P G L(2, L)$ of $G L(2, L)$ under the map (5) is called projective linear group in 2 variables over $L$.

The homomorphism $h_{J}$, (4), induces a homomorphism of $P G L(2, L)$ into $P G L(2, L / J)$ which we again denote by $h_{J}$. The determinant induces a map: $P G L(2, L) \rightarrow L^{*} / L^{* 2}$ which we again denote by det. Then the images $P G C(2, L, J)$ and $P S C(2, L, J)$ of the groups $G C(2, L, J)$ and $S C(2, L, J)$, respectively, under the map $P$, (5), can be characterized as follows:

$P G C(2, L, J)$

= group of the $\sigma \in P G L(2, L)$ with $h_{J} \sigma=1 \in P G L(2, L / J)$, $\operatorname{PSC}(2, L, J)$

$=$ group of the $\sigma \in P G L(2, L)$ with $h_{J} \sigma=1$ and $\operatorname{det} \sigma=L^{* 2}$.

In [5] we have determined the structure of the group $G L(2, L)$. Together with the preceding remarks, this yields the following

Structure Theorem for $P G L(2, L)$.

(i) Each subgroup $G$ of $P G L(2, L)$ which is invariant under $P S L(2, L)$ determines an ideal $J$ of $L$ such that

$$
P S C(2, L, J) \subset G \subset P G C(2, L, J)
$$

and, conversely, each subgroup $G$ of $P G L(2, L)$ satisfying (*) is invariant in $P G L(2, L)$.

(ii) In $P S L(2, L)$ all the invariant subgroups are of the form $P S C(2, L, J)$, where $J$ runs through the ideals of $L$. 
5. The isomorphisms of certain orthogonal groups over $V$ with projective linear groups in 2 variables, for $\operatorname{dim} V=3$ and 4 . The properties of the Clifford algebra $C(V)$ over $V$ yield in a natural way an isomorphism of certain orthogonal groups over 3- and 4dimensional spaces into projective linear groups in 2 variables. These isomorphisms are of fundamental importance for the determination of the structure of orthogonal groups of spaces with arbitrary dimension, cf. $\S 6$.

\section{Theorem 5.}

(i) Assume $\operatorname{dim} V=3$ and ind $V=1$ and $L / I \neq F_{3}$. Then $S O(V)$ is isomorphic to $P G L(2, L)$. Under this isomorphism, the group $S O(V, J)$ goes into $P G C(2, L, J)$ and the group $\Omega(V, J)$ goes into $P S C(2, L, J)$. In particular, $\boldsymbol{\Omega}(V)$ goes into $\operatorname{PSL}(2, L)$.

(ii) Assume $\operatorname{dim} V=4$ and ind $V=$ ind $g_{I} V=1$. Then $P S O(V)$ $=S O(V) /$ center $S O(V)$ is isomorphic to a subgroup $P U\left(2, L^{\prime}\right)$ of $P G L\left(2, L^{\prime}\right)$, where $L^{\prime}=L\left(\Delta^{1 / 2}\right)$ is a local ring, obtained from $L$ by a quadratic extension with an element $\Delta \in \theta(-1)$. Under this isomorphism, the group $P \boldsymbol{\Omega}(V, J)$, isomorphic to $\mathbf{\Omega}(V, J)$, goes into $P S C\left(2, L^{\prime}, J^{\prime}\right)$, $J^{\prime}=J L^{\prime}$. In particular, $P \boldsymbol{\Omega}(V)$, isomorphic to $\mathbf{\Omega}(V)$, goes into $P S L(2$, $\left.L^{\prime}\right)$.

6. The structure of the groups $S O(V)$ and $\Omega(V)$ for ind $V \geqq 1$. Let $G$ be a subgroup of $S O(V)$. The order of $G, o(G)$, is the smallest ideal $J$ with $S O(V, J) \supset G$, i.e., the smallest ideal $J$ with $h_{J} G \subset$ center $S O\left(g_{J} V\right)$.

Assume ind $V \geqq 1$. From Theorem 3 we see that $\Omega(V, J)$ has order $J$. From Theorem 4 (ii) we have that $S O(V, J) / \boldsymbol{\Omega}(V, J)$ is commutative, and since $\boldsymbol{S O}(V, J)$ and $\boldsymbol{\Omega}(V, J)$ are invariant, we have: Each subgroup $G$ of $\boldsymbol{S O}(V)$ satisfying $\boldsymbol{\Omega}(V, J) \subset G \subset \boldsymbol{S O}(V, J)$ is invariant and of order $J$. The following theorem asserts that these are the only invariant subgroups (and even the only subgroups invariant under $\boldsymbol{\Omega}(V))$ of $\boldsymbol{S O}(V)$ of order $J$ :

THEOREM 6. Let $V$ be a space over a local ring $L$, char $L / I \neq 2$. Assume ind $V \geqq 1, \operatorname{dim} V \geqq 3$. If $\operatorname{dim} V=3$, assume $L / I \neq F_{3}$. If $\operatorname{dim} V$ $=4$, assume ind $g_{I} V=1$.

(i) Each subgroup $G$ of $S O(V)$ of order $o(G)=J$ which is invariant under $\boldsymbol{\Omega}(V)$ satisfies the conditions

$$
\boldsymbol{\Omega}(V, J) \subset G \subset \mathbf{S O}(V, J) .
$$

Conversely, every subgroup $G$ of $S O(V)$ satisfying $\left({ }^{*}\right)$ is invariant in $\mathrm{SO}(V)$ and of order $J$. 
(ii) Each invariant subgroup $G$ of $\Omega(V)$ of order $o(G)=J$ is of the form $G=\mathbf{\Omega}(V, J)$ or $\mathbf{S O}(V, J) \cap \mathbf{\Omega}(V)$. $(\boldsymbol{S O}(V, J) \cap \boldsymbol{\Omega}(V)) / \boldsymbol{\Omega}(V, J)$ is isomorphic to center $\mathbf{\Omega}\left(g_{J} V\right)$.

REMARK. The proof of the preceding results is based upon an elaboration of the methods which have been developed, for an essential part by Dieudonné, for the investigation of the orthogonal groups over a field, cf. also Artin [1].

In particular, Theorem 6 is proved by relating the structure of the group $S O(V)$ with the structure of the groups mentioned in Theorem 5, and the structure of these latter groups is known, as we have stated in $\$ 4$, due to the results of our earlier paper [5].

\section{REFERENCES}

1. E. Artin, Geometric algebra, New York, Interscience, 1957.

2. N. Bourbaki, Algèbre, Paris, Hermann, 1959, Chapter 9.

3. J. Dieudonné, Sur les groupes classiques, Paris, Hermann, 1948.

4. - La géométrie des groupes classiques, Berlin, Springer-Verlag, 1955.

5. W. Klingenberg, Linear groups over local rings, Bull. Amer. Math. Soc. vol. 66 (1960) pp. 294-296.

\section{Mathematisches Institut, Göttingen, Germany}

